\title{
CIFAR: TRATAMIENTO TEXTUAL
}

Durante la década de los setenta, numerosos críticos e investigadores (destacan J. F. Burke, F. J. Hernández, R. Keightley, R. M. Walker) dedicaron su atención al Libro del Cauallero Zifar, intentando dilucidar problemas que, sin nuevas pruebas documentales, aún siguen irresueltos. El desconocimiento del autor, del lugar y fecha de composición; las dudas, incluso, sobre si es una "traslación" o una obra original en castellano; las diversas teorías - contradictorias casi siempre- sobre la estructura textual; las opiniones contrarias acerca de la unidad del Cifar, han formado un mosaico de aproximaciones y tanteos que, al menos, han despertado el interés por texto tan valiosísimo de la prosa literaria del s. XIV ${ }^{1}$. Faltaba acercarlo al lector: desde la edición de 1929 de Charles $\mathrm{Ph}$. Wagner ${ }^{2}$ ningún planteamiento ecdótico se había vuelto

1 Triste panorama el ofrecido por la labor editorial y divulgadora de estas primeras manifestaciones prosísticas. Sólo el Conde Lucanor de don Juan Manuel y alguna obra del período alfonsí han merecido la atención de ser presentadas al público en tiradas lo suficientemente amplias como para asegurar su extensión. Pero, por ejemplo, las creaciones de López de Ayala, la literatura de los 'exempla' y las obras cronísticas son inencontrables en ediciones orientadas a un gran público. Es desolador que muchas de estas obras sólo se conserven como reşultado de trabajos de investigación universitaria, desarrollados fuera de España y sólo localizables en muy pocas bibliotecas. Al menos, queda el recurso de acudir al manuscrito original, si no ha desaparecido en cualquier evento histórico.

2 Publicada en Ann Arbor, University of Michigan, 1929; con reimpresión en Milwood-Nueva York, Kraus, 1972. J. González Muela -que adelanta equivocadamente el año de impresión a 1912- resume sus defectos: «mezclar los dos manus- 
a orientar al estudio de las tres versiones antiguas conservadas: $M$ y $P$, manuscritos de los ss. XIV y xv, y $S$, impresión de Sevilla de $1512^{3}$.

Ahora, en sólo tres años, este vacío textual se ha colmado con tres nuevas ediciones ${ }^{4}$ de muy distinta índole que facilitarán la lectura de esta obra, antes accesible sólo a un estrecho círculo de especialistas. Complemento de estas aportaciones es el libro de Cristina González, "El Cauallero Zifar» y el reino lejano ${ }^{5}$, en el que propone otra interpretación de la estructura textual.

Tal avalancha de ediciones es explicable por la revalorización de la obra conseguida por la crítica de los años setenta. Son múltiples los motivos para leer el Cifar. De entrada, la obra introduce al lector en una situación social llena de tensiones políticas, por las que la nobleza va adquiriendo un papel más predominante, que corresponde a la afirmación del caballero como elemento nuclear de una sociedad en crisis, pero en proceso de crear una cultura que sea reflejo de esos valores individuales, en cuyo esfuerzo se ha centrado la primera etapa de la Reconquista. Los estudios sociológicos han encuadrado la obra en las luchas internas habidas en el reinado de Sancho IV, en un momento histórico en que se crea la caballería de cuantía, poseedora de un determinado patrimonio que obliga a mantener caballo y armas ${ }^{6}$. Así enfocado, el Cifar deja ver cómo el hombre persigue su salvación, pero a través de una concepción digna y profunda de lo que debe ser la vida de un caballero; como se lee en el Prólogo, el individuo posee una estimación intelectual de sí mismo (orientada en un sentido religioso):

E por ende todo ome que alguna cosa o obra buena quiere començar deve anteponer en ellas a Dios; e él es fazedor e mantenedor de las cosas, así puede bien acabar lo que començare, mayormente si buen seso natural toviere (ed. González Muela, pág. 57).

critos y la ed. impresa de Sevilla de 1512, suponiendo que las versiones más modernas llenan las 'lagunas' del Ms. M, el más antiguo», ver ed. de González Muela, pág. 45.

3 Las ediciones de Martín de Riquer, Barcelona, Sel. Bibliofilas, 1951 y de Felicidad Buendía, Madrid, Aguilar, 1954, reproducen la de Wagner, modernizando el texto.

4 Por orden: 1.") Libro del Caballero Zifar, ed. de Joaquín González Muela, Madrid, Castalia, 1982; 2.") Libro del Caballero Zifar, ed. de Cristina González, Madrid, Cátedra, 1983; 3.") Libro del Cauallero Çifar, ed. de Marilyn A. Olsen, Madison, The Hispanic Seminary of Medieval Studies, 1984.

5 Madrid, Gredos, 1984.

6 Es importante hacer notar que este desarrollo ya se había producido en la Francia de la segunda mitad del s. xir donde la clase noble de la caballería buscó una peculiar expresión artística, que le fuera propia y le dotara de prestigio. Esto no se produce en España hasta que Alfonso $\mathrm{X}$ regula, en las Partidas, las normas de convivencia de su corte. El hecho de que un noble se convierta en paradigma cultural no se encontrará hasta el s. xiv (iy aún don Juan Manuel debe defenderse de los que critican su labor literaria!). 
De aquí surge una voluntad de reflexión y de conocimiento humano, que se constituye en modelo por el que los personajes de esta obra van a existir y a crear una representación de una vida conforme a unas características previas desde las que se definirán; así, por ejemplo, Cifar era un caballero "complido de buen seso natural», que "nunca despero de la merçed de Dios», y su mujer Grima "fue muy buena dueña e muy mandada a su marido e mantenedora e guardadora de la su casa». Se crean caracteres modeladores de una realidad que, de otra forma, no se hubiera podido conocer.

Pero lo importante es que el Cifar sea una obra de ficción, y si existe esa articulación histórica y social no es más que para levantar sobre ella un argumento que demuestre los propósitos didácticos iniciales del autor: el hombre de buen seso natural debe siempre realizar buenas y honestas obras a servicio de Dios, ya que así, pese a los «enbargos» y dificultades de la vida, alcanzará un final adecuado a su posición humana y social. De este modo, el Cifar es una historia en que se cuenta cómo un caballero parte de unas condiciones negativas ("pero atan fuerte era la fortuna del marido»), desarrolla una vida adversa («pasando primeramente por muy grandes trabajos e grandes peligros $*$ ), concluyendo en una situación próspera y dichosa para toda su familia. La lectura adquiere, por ello, su mayor interés al constituirse en canal de unos hechos argumentales que reúnen informaciones de toda clase: folklórica, hagiográfica, legendaria "exemplos», siendo el libro un punto de reunión de las tres culturas, cuya convivencia había promovido el Rey Sabio.

En esta perspectiva, editar el Cifar era totalmente necesario; ahora bien, como se ha señalado, el valor de las tres ediciones es muy diferente; a fin de determinarlo, marco los siguientes principios como criterios de juicio:

\section{Trabajo textual}

Si se acepta que la edición de Wagner (ver nota 2) no es crítica, ninguna de estas tres lo es; en primer lugar porque dos de ellas transcriben sólo un manuscrito: González Muela el $M$ (Bibl. Nac. de Madrid, $M s$. 11309; de muy difícil lectura) y Marilyn A. Olsen el $P$ (Bibl. Nac. de París, Esp. 36; joya bibliográfica por sus 242 miniaturas); ni presentan registro de variantes ni se comparan los manuscritos, en parte porque no es intención de los editores ?. Por otro lado, C. González confiesa

7 Señala González Muela. «Publicamos, pues, la versión del Ms. $M(\ldots)$ y esperamos que pronto aparezca una edición del $M s$. $P$, con la que el nutrido grupo de investigadores que hoy se dedica a seguir las pistas del Zifar podrá trabajar sobre 
reproducir el texto de Wagner, eliminando las variantes ${ }^{8}$. Quiere decirse, entonces, que se cuenta con una nueva reimpresión del texto fijado por Wagner en 1929, y con dos transcripciones de los manuscritos conservados, ambas muy bien efectuadas.

He realizado algunas calas comparando la transcripción de Muela con el códice de la Bibl. Nac. de Madrid, sin observar ninguna alteración, pese a la dificultad de la letra del manuscrito, pero en cambio la lectura del Ms. $P$-con la que González Muela, igual que Wagner hizo, rellena las lagunas de $M$ - sí es defectuosa; dejando de lado la modernización de grafías, con la puntuación y acentuación actuales, Muela corrige el manuscrito sin indicarlo, igual que cuando omite palabras del mismo; es más exacta, en este sentido, la transcripción de Wagner, aunque la versión modélica por su perfección sea la presentada por Marilyn A. Olsen $^{9}$. Para comprobar estas diferencias, he cotejado el folio 1 del Ms. $P$ (reproducido en la pág. 50 de la edición de Muela) con las lecturas de los tres críticos, observando los siguientes hechos:

a) Muela conserva la lectura de Wagner, quien modificó el texto del manuscrito sin idicarlo; Olsen ofrece la lectura correcta.

- Ms. P, col. I, lín. 2-4: «bonjfaçio vjno enla era de mjll e trezientos años el dia de la naçençia de n[uest]ro Señor ih[es]u xp̄o començo en[e]l año jubileo».

- ed. Wagner, pág. 1, lín. 1-3: «Bonifaçio VIII', en la era de mill e trezientos años, en el dia de la naçençia de Nuestro Señor Iesu Cristo, començo el año jubileo».

—ed. Muela, pág. 51, lín. 1-3: «Bonifaçio VIII, en la era de mill e trezientos años, en el día de la naçençia de nuestro señor Ihesu Christo, començó el año jubileox.

- ed. Olsen, pág. 1a, lín. 1-3: «Bonjfaçio, vjno en la era de mjll e trezientos años el dia de la naçençia de nuestro Señor Ihesu Xpisto, començo en el año jubileox.

una base solida», ver ed. cit., págs. 46-47; MARILYN A. OLSEN, «The purpose of this edition is to provide an accurate transcription of MS $P_{x}$, ver ed. cit., pág. XIV, $y$ rechaza la edición de Gonzalez MUELA, «Who primarily follows Wagner, but with some alterations», ibidem, n. 3, pág. XVII.

8 «La presente edición sigue el texto de la edición de Wagner, a la cual se le ha suprimido el aparato crítico», ver ed. cit., pág. 58.

9 No en vano lleva trabajando en el proyecto desde 1970; se doctoró con una tesis sobre este tema: The Manuscripts, The Wagner Edition, and The Prologue of the Cauallero Zifar (Tesis doctoral, Universidad de Wisconsin en Madison, 1975); adelantó algunas cuestiones en «Three observations on the Zifar», en La Corónica, 8 (1980), págs. 146-148 y en aA Reappraisal of Methodology in Medieval Editions: The Extant Material of the Libro del cauallero Zifar», en Romance Philology, 35 (1982), páginas 508-515. 
(hay, pues, una corrección del ms. que tiende a ofrecer una versión más lógica de su significado, alterando para ello el orden de los conglomerados preposicionales; la solución es positiva, aunque no se ha dejado constancia de la enmienda. Olsen prefiere dar la lectura del ms. a interpretarla, por eso ella conserva «vino» en vez del «VIII ${ }^{\star}$ de los otros editores).

b) Muela difiere del manuscrito, reproducido con fidelidad en Wagner y en Olsen.

- Ms $P$, col. I, lín. 18-19: «auja enlas yndulgençias deste año al jubileo e alos enojos....

- ed. Wagner, pág. 1, lín. 13: «auia en las yndulgençias deste año al jubileo, e a los enojos.....

- ed. Muela, pág. 51, lín. 14-15: «avía en las indulgençias de este año jubileo, e a los enojos...».

- ed. Olsen, pág. 1a, lín. 13-14: «auja en las yndulgençias deste año al jubileo e a los enojos.....

(aparece suprimida la contracción «al» en Muela, lo que puede mejorar el sentido de la frase, pero elimina la posibilidad de existencia de un hipérbaton: «auia en las yndulgençias [al jubileo] deste año», lo que implicaría eliminar la conciencia creativa del autor).

c) Muela cambia la construcción preposicional, en contra del manuscrito, de Wagner y de Olsen.

- Ms. $P$, col. II, lín. 35-37: «E otrosi alos que començaron el camjno para yr en [e]sta rromeriaw.

- ed. Wagner, pág. 1, lín. 25-26: «e otrosi a los que comen[c]aron el camino para yr en esta romeria".

- ed. Muela, pág. 52, lín. 2-3: «e otrosí a los que començaron el camino para ir a esta romeríaw.

- ed. Olsen, pág. 1a, lín. 26-27: «e otrosi a los que començaron el camjno para yr en esta rromeria».

(Muela elimina, de este modo, el sentido temporal de la preposición «en», que encuadra mejor en la información general proporcionada por el texto).

d) Wagner corrige el manuscrito indicándolo, mientras que Muela elude esta función; Olsen se limita a transcribir el texto.

- Ms. P, col. II, lín. 51-53: «Et fue y despendido el poder del padre santo catodos aquellos clerigos que cayeron en yerro [...] yrregularidat non vsando de sus ofiçios...». 
- ed. Wagner, pág. 2, lín. 7-10: «E fue y despendido el poder del Padre Santo contra todos aquellos clerigos que cayeron en yerro [o] yrregularidat, non vsando de sus ofiçios...».

- ed. Muela, pág. 52, lín. 14-16: «E fue ý despendido el poder del Padre Santo contra todos aquellos clérigos que cayeron en yerro o irregularidat non usando de sus ofiçios...».

- ed. Olsen, pág. 1a, lín. 37-39: «E fue y despendido el poder del padre santo ca todos aquellos clerigos que cayeron en yerro [...] yrregularidat, non vsando de sus ofiçios...».

(la corrección de los dos críticos mejora la lectura, ya que se apoya en un sintagma paralelo posterior: «E fue despendido contra todos aquellos...», lo que permite suponer que el copista o no resolvió la abreviatura o se equivocó; ante ello, Wagner sí indica su alteración: «8 contra]ca», lo mismo hace al incluir la conjunción disyuntiva «o» que Muela incorpora a su texto sin más señalización).

\section{Puntuación}

Desde que el Seminario de Estudios Medievales, dirigido por J. Roudil en la Universidad XIII-París, ha levantado la sospecha de que en los manuscritos medievales pudiera existir otro sistema de puntuacion distinto al nuestro, se debe valorar con sumo cuidado cualquier trabajo que en esta dirección se emprenda; Muela sólo indica: «La puntuación es nuestra» (pág. 46), y adoptar una responsabilidad de este hecho es muy grave, ya que se debe tener en cuenta que nuestra concepción de la frase nada tiene que ver con la medieval (incluso el aparato sígnico). De todos modos, no deben tolerarse en una modernización de la puntuación incoherencias de sentido, surgidas de una falta de sistematización del método empleado, así se puede leer en la pág. 203:

*Amigos -dixo Roboán-, podremos oir misa en este canpo ante que lleguen aquellos cavalleros; ca en todos los nuestros fechos devemos anteponer a Dios.» «Señor - dixo un capellán-, muy bien la podedes oir, ca yo vos diré misa privadax.

(las comas después del guión están bien, pero hay un desajuste sintáctico al encontrar delante del primer «ca» un punto y coma y en el segundo una coma, existiendo en ambos un matiz consecutivo).

Esto puede dar lugar a ambiguiedades si estos signos de puntuación se colocan sin rigor:

«E el ribaldo se fue para el hermitaño e non falló $y$ al cavallero nin al hermitaño, e fuese para la villa e fallólos que óan misa. El cavallero quando lo vio plógole, e díxole...* (pág. 141). 
(en el último caso sobra la coma ya que, como antes se marcó, dos estructuras sintácticas coincidentes pueden muy bien ir unidas por la propia conjunción copulativa).

Hay algún caso en que se plantean problemas de comprensión, originados por una mala disposición de estos signos:

«E acaesçióles una noche de albergar en una alberguería do yazían dos malos omes ladrones e andavan en manera de pelegrinos, e cuidaron...» (id.)

(sólo hay una coma que separa la posible confusión de sujetos - Cifar y Ribaldo frente a los dos ladrones- pero para que hubiera sido más efectiva tendría que haberse colocado en el período anterior: «omes ladrones, e andavan...», ya que ahí sí existe un cambio de disposición estructural).

No se pretende responsabilizar a Muela de estos errores, ya que en líneas generales sí existe una correcta interpretación del significado textual, pero es conveniente volver a llamar la atención sobre el hecho de que una edición de un texto medieval debe pasar por una profunda reflexión acerca de la concepción de los espacios textuales que ese manuscrito deje percibir ${ }^{10}$.

Para Cristina González el problema de la puntuación no existe: reproduce el sugerido por Wagner.

Sólo Marilyn A. Olsen detiene su atención en este aspecto; al menos da cuenta de las marcas que el propio manuscrito presenta ", aunque luego acomode el texto medieval a las normas modernas, con una correcta adecuación sígnica y sintáctica; prefiere la editora frases breves y concisas separadas por punto y seguido, que no perderse en la farragosidad de comas que compliquen el sentido de la frase.

\section{Criterios de edición}

González Muela conserva las grafías con valor fonológico y, en los demás problemas (unión y separación de palabras, acentuación, abreviaturas), procede según normas modernas.

\footnotetext{
10 El primer trabajo serio sobre este tema lo planteo JEAN RoubIL, «Edition de texte, analyse textuelle et pontuaction. (Brèves réflexions sur les écrits en prose)», en Cahiers de linguistique hispanique médiévale, 3 (1978), págs. 269-299. Esta misma revista ha dedicado a estas cuestiones un número monográfico: ver 7 bis (1982).

11 *Regarding the punctuation, my analysis reveals that the few marks which might be interpreted as punctuation are primarily line fillers. They consist of a slash with a dot underneath (/.), and they are essentially limited to the last ten folios», ver ed. cit., pág. XIV.
}

LXVI, 3.0-4.o. - 10 
Resulta obvio que Cristina González no altere nada de lo hecho por Wagner (sólo elimina la letra bastardilla y las indicaciones de los folios del manuscrito).

Es, de nuevo, Marilyn A. Olsen quien actúa con más rigor para acercar al lector moderno el texto tal como se conserva en el Ms. P: para ello, hay una total conservación de grafías, y no porque quiera ofrecer una versión paleográfica, sino porque quiere respetar en sí la forma textual; Olsen, por ejemplo, percibe formas dialectales: en su edición se conservan inalteradas por la sospecha de que la obra pudiera ser una «traslación».

En cuanto a la división por partes y capítulos, González Muela y Cristina González conservan la concepción estructural de cuatro amplios planos temáticos sugerida por Wagner; por su parte, Olsen no ha dividido el texto, aunque ofrece algunas indicaciones a fin de poder seguir la línea argumental.

\section{Aparato crítico}

González Muela ofrece muy pocas notas a pie de página para la cantidad de problemas de toda índole que el Cifar plantea. Apenas se aclaran las dificultades textuales dimanadas de la lectura del manuscrito (y si se hace, es remitiendo a la edición de Wagner; así, se lee, por ejemplo, en la nota 43: «El caballero promete darle dos hijas para que se casen con los dos fijuelos [En el texto de $W$. .)); por supuesto, las correcciones y enmiendas de Muela se omiten también; por ello, se pueden hallar incomprensiones que deberían haber sido explicadas en nota, de este modo realizando una cala en una misma página (166) se encuentra:

- «que si do dos mill cavalleros toviese, non más atreverme ía a entrar» (ese «do», ¿es «dónde»?, ¿qué sentido tiene?, ¿es errata?).

- «eñor, tú sabes que muchos condes e muchos omes buenos de alta sangre fueron aquí venidos para te servir, e demás pára mientes quién das tu fija» («pára», cacentuado así tiene algún significado?, ¿por qué no se indica?).

Esta carencia de aclaraciones no queda justificada por una nota previa: «El lector encontrará en esta edición frases o párrafos de difícil comprensión. En algunas notas, los hemos aclarado, pero en muchos casos creemos que no podemos ni debemos corregir nada" (pág. 46); es evidente que no se piden correcciones, aunque sí explicaciones, que es el requisito mínimo de una edición anotada. 
Los contenidos linguísticos son también insuficientes, cuando un análisis sintáctico o morfológico puede aclarar las cuestiones referentes, por ejemplo, a los débitos culturales de la obra. Unnicamente se acompaña al final de la edición un glosario, de cuyos términos se podía haber indicado la evolución etimológica (este vocabulario reproduce, de todos modos, las notas que a pie de página ya habían indicado esos significados).

Existen pocas referencias a los problemas sociológicos e históricos que son muchos y variados, en todo caso se remite a la Introducción; sí es acertada la inclusión de trabajos particulares sobre el Cifar, llegado el momento textual en el que la cuestión determinada suscitó el estudio (así, las $\mathrm{nn} .260,264,281 \ldots$ ); en este sentido, también pueden encontrarse autores medievales, citados con el fin de verificar un paralelo de términos linguísticos.

Frente a las 282 notas de González Muela, Cristina González acompaña a su edición de 552 referencias: el escaso trabajo textual se ve compensado por esta mayor riqueza informativa, ya que aparte de las explicaciones lingüísticas y sintácticas, esta editora se detiene mucho en analizar los procedimientos narrativos que se plantean en el Cifar; hay ocasiones, incluso, en que dibuja (n. 419) un esquema estructural para mostrar la articulación de unos enxiemplos; en otros lugares, no duda en citar obras y autores que han abordado esos problemas (ver, por ejemplo, nn. 358, 371, 430, 480, etc.); son, también, frecuentes las alusiones internas a motivos y aspectos temáticos de la propia obra, que muestran la unidad estructural del texto, con sus paralelismos y contrastes.

Por su parte, la edición de M. A. Olsen sólo lleva 35 notas (pág. 153) referidas, en exclusividad, a problemas textuales (correcciones de la editora).

\section{Introducción crítica}

En el caso de la edición de González Muela, quizá debido al propósito divulgador de la misma o a un corto número de páginas permitidas a este aspecto, la "Introducción crítica» resulta lo más pobre e insustancial del libro. Bien que se tratan los problemas fundamentales, desde la especificidad de la obra hasta su estructura y lenguaje, bien que Muela busca utilizar el texto como elemento central de su explicación, pero lo que no resulta lógico es construir una visión general del Cifar mezclando y parafraseando estudios y artículos publicados sobre esta 
obra, por muy importantes que sean, cuando aún existe una gran cantidad de cuestiones no abordadas.

Comienza con un argumento global de la obra, para intentar luego enfocarla genéricamente, empleando el nombre de «novela de caballerías ${ }^{12}$. A partir de aquí se introduce en el problema de tratamiento de las aventuras en el libro; para Muela el primer punto es un canto de exilio, que define unas condiciones adversas de las que parte un caballero y que podrían estar apuntadas en el prólogo, ya que éste posee un protagonista independiente - Ferrán Martínez, arcediano- que emprende una especie de demanda caballeresca. Al tratar la cuestión de la autoría, Muela cree que este arcediano es el creador del Cifar y para ello lo sitúa en una perspectiva histórica, ya marcada por los brillantes trabajos de F. J. Hernández sobre este punto; se hace un amplio resumen de los mismos, de lo que lo más destacado es la relación entre experiencias personales de F. Martínez y capítulos enteros del Cifar (de ahí, la alusión a los problemas habidos en el reinado de Sancho IV).

El resto de la Introducción se articula desde los trabajos de Walker y Burke en cuanto a los problemas de fechación de la obra y de sus fuentes (ocasión ésta desaprovechada para intentar explicar el funcionamiento de los materiales folklóricos; sobran las manidas referencias a los dos artículos de Krappe de los años treinta, uno de ellos refutado por Lida).

Los problemas de la estructura y del lenguaje reciben también una atención mínima: si bien se alude a la cuestión del sermón universitario - pensado por Burke- como punto central desde el que se realiza una "amplificatio» constructiva de los temas, no se llega a explicar el sentido interno que hilvana los episodios de la obra (sólo se menciona que Keightley estudia este punto).

Por último es sugerente una comparación entre el estilo del Conde Lucanor (plano más distante del lector) y el del Cifar (economía de palabras) sobre un episodio parecido al de ambas obras.

Cristina González presenta una «Introducción» más homogénea y profunda que la de González Muela. En principio, ella encuadra la obra

12 Dejando de lado el hecho de que esta denominación no debe aplicarse a estas obras prosísticas de finales del s. xIII y comienzos del s. XIV, y menos al Cifar como ya demostró Luciana di STÉfano, "El Caballero Cifar", novela didáctico-moral», en Thesaurus, 27 (1972), págs. 173-260, lo menos que se podía haber esperado es una definición que justificara el empleo que Muela realiza de este término, y en vez de ello, se limita a reducir la cuestión a una amplia exposición de su materia. Estos estudios deben comenzar por la limitación de que no se pueden aplicar terminologías genéricas a conceptos culturales no existentes en una época determinada. 
en el género del «libro de aventuras» ${ }^{13}$, aunque indicando que su especificidad radica en constituir una 'historia de familia separada y reunida', caso también del Plácidas.

Aborda, luego, el problema de las fuentes, planteando un resumen del estado de la cuestión (Wagner, Krappe, Scudieri, Walker, Riquer), para indicar - lo más discutible de la Introducción- que «o bien el Zifar copia las verdaderas traducciones, o bien es una auténtica traducción. Puesto que en la Edad Media las obras que se declaraban traducciones solían serlo, podemos asumir que el Zifar es una verdadera traducción" (pág. 19); como señala Riquer, este recurso de la 'traslación' es un tópico de la materia caballeresca, en confirmación de esto, recuérdese que se repite la misma idea al final de "Los hechos de Roboan", quien tuvo un hijo «que ouo nonbre Fijo de Bendiçion, asy commo ya oyestes, de que dizen que ay fecho vn libro en caldeo, en que cuenta toda la su vida e muchos buenos fechos que fizo» (ed. de C. González, pág. 457).

Sobre los problemas de la fecha y el autor, continúa resumiendo las aportaciones de otros críticos; igual hace en el capítulo del estilo, aunque ahora añade unas comparaciones entre fragmentos de la Estoria de España, del Cifar y del Conde Lucanor, al final concluye: "el Zifar emerge como una obra que, frente a la ausencia de estilo, personal de la Estoria de España, posee un estilo propio, y que, frente a la presencia de estilo personal de El Conde Lucanor, posee un estilo personal diferenciado» (pág. 31).

El modelo estructural es más sugerente y rico en perspectivas; C. González resume las aportaciones de la crítica: Burke (habló de 'alegoría de los poetas'), Walker (basado en el recurso de la amplificatio de las artes poeticae), F. J. Hernández (centrado en la magnificentia, tema central de la alegoría) y $\mathbf{M}$. Ana Diz (los "Castigos del rey de Mentón» es la parte más significativa, las otras son exempla). C. González propone otra vía de análisis: los libros de aventuras no suelen tener un significado alegórico continuo; para ella, «el Zifar contiene exempla, pero no es un exemplum, es un libro de aventuras medieval y, como tal, no demasiado complicado" (pág. 38). Tiene mucha razón cuando indica que estos libros combinan elementos narrativos y didácticos, sin que predomine uno u otro plano; así, el Cifar no tiene que concebirse como una enseñanza verdadera ilustrada por historias falsas,

13 Nombre propuesto por Deyermond, a fin de rellenar el vacío terminológico causado por la carencia en español del vocablo roman para designar la obra en que la aventura es el eje estructural. 
sino como una historia falsa aderezada por enseñanzas verdaderas. Queda claro, pues, que la unidad debe buscarse en el «plano real», formado por la estructura argumental de los personajes; ésta se divide en dos secciones: I. Aventuras de Cifar (de carácter ficticio): a) Introducción: caps. 2-10, trata de la partida; b) Conquista del reino: caps. 11-121. II. Aventuras de Roboán: a) Introducción: caps. 122-178, trata de la partida; b) Conquista del imperio: caps. 179-229. Aspecto original es buscar en el Prólogo la misma división estructural: I. Aventura de Ferrán Martínez (de carácter real): a) Introducción; b) Traslado del cuerpo. II. Aventura del trasladador (de carácter literario): a) Introducción; b) Traslado de la obra.

En el último apartado, revisa las distintas denominaciones genéricas que se han aplicado al Cifar; corrige el modelo de Armando Durán, Estructura y técnicas de la novela sentimental y caballeresca, y señala que, en el grupo de los libros de aventuras caballerescas, habría que considerar una primera fase de iniciación-transformación (donde se encuadraría el Cifar) y otra segunda de transformación-repetición (Amadis).

Concluye afirmando que el Cifar es una novela de caballerías típica de la Edad Media castellana, con un mundo donde el ascenso es posible no sólo para los caballeros sino también para los siervos.

La «Introduction» de Marilyn A. Olsen es muy breve; reducida, sobre todo, a plantear los problemas ecdóticos con los que se ha enfrentado en el texto (desde aceptar el método del filólogo alemán Lachmann hasta seguir el "pecia system"). Indica que $M, P$ y $S$ no proceden de un mismo original y que por ello conviene publicarlos por separado.

Sobre el lugar de composición, fecha y autor no añade nada nuevo; resume el estado de la cuestión y se pregunta cómo Ferrand Martines - si es verdad que fue el autor de la obra- olvidó la verdadera fecha del fallecimiento (1299) del cardenal Gonzalo Gudiel.

Describe, después, el manuscrito $P$ y señala, meticulosamente, los criterios de edición empleados.

\section{Bibliografia}

En la edición de González Muela, faltan algunos artículos importantes de Blüher, varios aspectos episódicos estudiados por Burke y no contenidos en su libro, los puntos estructurales señalados por Marta Ana Diz, algún artículo de F. J. Hernández sobre la alegoría y el propio Ferrán Martínez, el muy importante de Scudieri Ruggieri sobre el 'ribaldo' y aquél en el que Walker estudia la unidad de la obra. 
Cristina González y Marilyn A. Olsen ofrecen una bibliografía más completa y actualizada. De la primera sólo echo de menos un artículo de Levi (1933-34) y los dos clásicos estudios de Erich von Richtofen. De la segunda nada hay que reprochar ${ }^{14}$; al contrario, es la más perfecta: en ella se encuentran citadas todas las tesis doctorales inéditas realizadas en las Universidades americanas y se adjuntan las reseñas críticas elaboradas sobre los libros en que se ha estudiado el Cifar.

Como conclusión, se puede afirmar que la mejor edición, por el trabajo textual, es la de Marilyn A. Olsen; la de Cristina González destaca por su Introducción y su aparato crítico, mientras que la de González Muela sobresale por ofrecer una lectura correcta del difícil Ms. $M$.

Cristina González amplió la visión crítica del Cifar con un posterior libro titulado "El Cavallero Zifar" y el reino lejano ${ }^{15}$, donde se encuentran ampliadas las aportaciones más originales que la autora desglosó en su edición. Continúa con el mismo sistema que allí empleó: resumen de las aportaciones críticas y punto de vista suyo. Lo positivo de este procedimiento estriba en que facilita mucho el acercamiento a la obra encontrar condensados los estudios más interesantes que se han dedicado a ella; pero resulta desmesurado dedicar más de la mitad de las 150 páginas de que consta el libro a ofrecer lo que otros han pensado o descubierto, mermando así el espacio en que la autora debía haber expuesto sus ideas ${ }^{16}$, que son muy interesantes: un ejemplo, la separación como personajes diferentes de Ferrán Martínez y el trasladador.

Los capítulos más importantes son el IV (estructura y significación) y el V (problema del realismo de la obra). La estructura es enfocada desde la siguiente perspectiva: "El Zifar es una novela medieval y, como tal, contiene otros géneros literarios y/o presenta algunas características de ellos» (pág. 78). Señala después que "el deseo es el motor narrativo» (pág. 89), siendo esa idea de mejoramiento la determinadora de la estructura de la narración ${ }^{17}$.

El capítulo $\mathrm{V}$ es el que presta el título al libro: analiza en él los episodios maravillosos del Cavallero Atrevido y de las Ynsulas Dotadas,

14 Marilyn A. Olsen ya publicó una *Tentative Bibliography of the "Libro del Cauallero Zifar"», en La Corónica, 11 (1983), págs. 327-335.

15 Madrid, Gredos, 1984; es curioso que en su bibliografía de Cátedra el título sea otro: Aproximación al «Libro del Cavallero Zifar».

16 Como en el caso de M. A. Olsen y de F. J. Hernández la base de sus investigaciones se encuentra en su tesis doctoral: «Estructura y significación del "Libro del Cavallero Zifar"”, Diss. Indiana, 1981; DAI, 43 (1982-83), 165A.

$17 \mathrm{De}$ todos modos, los diseños estructurales ofrecidos en las págs. $40-45$ de su edición son más claros que los leves apuntes contenidos en la pág. 80 y en la pág. 92. 
señalando las similitudes de ambos (sus dos protagonistas se han enfrentado con las fuerzas del bien y del mal; los dos han regresado mediante una ruptura: el Cavallero Atrevido lo hace escarmentado y alegre, mientras que Roboan vuelve arrepentido y triste). Son aventuras en las que se ha viajado a un 'reino lejano': mundo fantástico donde se multiplican el tiempo y el espacio del 'reino cercano' (Mentón y Trigrida, que deben ganarse, frente a los otros que se habían perdido). Son episodios folklóricos, piensa $\mathrm{C}$. González, y no explicables desde la «alegoría de los poetas» como sugería Burke. Este es el análisis más satisfactorio del libro, aunque insuficiente, porque esa base tradicional no se explica, ni se muestra la construcción del relato desde ella.

El capítulo VI es un empeño por dilucidar unas mínimas nociones genéricas aplicables al Cifar; compara seis textos de materia caballeresca - dos castellanos, dos catalanes y dos portugueses ${ }^{18}$ - para indicar que hay una sutil transformación en el género, porque las obras que comienzan siendo un fenómeno medieval de defensa de los caballeros, acaban siendo un fenómeno moderno de defensa de los reyes.

En cierta manera, lo más coherente de este libro es su "Conclusión»: alli apunta una nueva idea original, apenas explicada, «el Zifar no tiene unidad, sino duplicidad” (pág. 133), por ser dos los protagonistas, Cifar y Roboan.

Incide, de nuevo, en su estructura de mejoramiento y en el modo en que el 'trasladador' - ser real y ficticio a la vez - conecta los dos mundos: el de F. Martínez y el de los héroes.

Por último, recuerda que la técnica de construcción de la obra es la concatenación y que la idea central del argumento es que «cualquiera puede ascender o descender; depende de su mériton.

En suma, un libro cuya utilidad se encuentra en ser auxiliar imprescindible para conocer el estado de la cuestión crítica sobre el Cifar y cuyas sugerencias pueden abrir nuevas vías de estudio e investigación ${ }^{19}$.

Fernando Gómez Redondo

18 Resulta muy grave cotejar obras no sólo alejadas diacrónicamente, sino desconectadas geográficamente entre sí; el perfil de un género literario debe buscarse en la génesis de una obra y en sus sucesiones, como plantea Curto Herrero para el Amadis, pero nunca pueden equipararse obras producidas durante más de dos siglos; aunque compartan la misma temática, sus motivos creadores son distintos.

19 Concluyo dando cuenta del último estudio aparecido sobre el Cifar: MichaEl HARNEY, *The Geography of the Caballero Zifar», en La Corónica, 11 (1983), páginas 208-219. Su visión puede contrastarse con la teoría de Cristina González sobre el 'reino lejano'. 